\title{
Investigation and control of a large outbreak of multi-drug resistant tuberculosis at a central Lisbon hospital
}

\author{
M. M. Hannan, H. Peres*, F. Maltez†, A. C. Haywardł, J Machadof, A. Morgadof, \\ R. Proenca†, M. R. Nelson, J. Bico*, D. B. Young§ and B. S. Gazzard
}

Department of Medical Microbiology and HIV/Genito-Urinary Medicine Unit, Chelsea and Westminster Hospital, 369 Fulham Rd, London UK; *Microbiology Department, and fInfection Disease Unit, Hospital Curry Cabral, Rua da Beneficiencia, 1000 Lisboa, Portugal; $\ddagger$ Public Health Laboratory Services, Communicable Disease Surveillance Centre, 6 I Colindale Avenue; and §Department of Infectious Diseases, Imperial College School of Medicine, Norfolk Place, London, UK

Summary: An increase in the number of new cases of tuberculosis (TB) combined with poor clinical outcome was identified among HIV-infected injecting drug users attending a large HIV unit in central Lisbon. A retrospective epidemiological and laboratory study was conducted to review all newly diagnosed cases of TB from 1995 to 1996 in the HIV unit. Results showed that from 1995 to 1996, 63\% (109/173) of the Mycobacterium tuberculosis isolates from HIV-infected patients were resistant to one or more anti-tuberculosis drugs; $89 \%$ (95) of these were multidrug-resistant, i.e., resistant to at least isoniazid and rifampicin. Eighty percent of the multidrug-resistant strains (MDR) available for restriction fragment length polymorphism (RFLP) DNA fingerprinting clustered into one of two large clusters. Epidemiological data support the conclusion that the transmission of MDR-TB occurred among HIV-infected injecting drug users exposed to infectious TB cases on open wards in the HIV unit. Improved infection control measures on the HIV unit and the use of empirical therapy with six drugs once patients were suspected to have TB, reduced the incidence of MDR-TB from 42\% of TB cases in 1996 to $11 \%$ in 1999.

(C) 200 I The Hospital Infection Society

Keywords: Multidrug-resistant tuberculosis; nosocomial transmission; HIV infected; DNA fingerprinting.

\section{Introduction}

The HIV/AIDS epidemic has had a major impact on the incidence of tuberculosis (TB) in many

Received 11 August 1999; revised manuscript accepted 18 October 2000

Author for correspondence: Dr M. Hannan,

CDC/NCHSTP, Division of TB Elimination, Surveillance and

Epidemiology Branch, 1600 Clifton Rd., Mail Stop E-10,

Atlanta GA 30333, USA. Fax: 404-639-8959.

Presented in part at the 7th International Congress for Infectious Diseases, Hong Kong, June 1996 and the European

Tuberculosis Society, Cordoba, Spain, June 1997.

Supported by St Stephen's Trust and the Wellcome Trust. countries. ${ }^{1}$ The areas most affected by the dual pandemic are Sub-Saharan Africa, Southeast Asia and, most recently, the Indian subcontinent. ${ }^{2}$ There have been increasing reports of drug resistant strains of Mycobacterium tuberculosis among HIV-infected patients and dramatic outbreaks of multidrug-resistant TB (MDR-TB) have been reported in HIV-infected patients in the United States $^{3-9}$ and Europe. ${ }^{10-13}$ We describe an outbreak of MDR-TB among HIV-infected injecting drug users attending a hospital in central Lisbon and discuss the measures used to reduce ongoing transmission. This outbreak is one of the largest documented nosocomial outbreaks of MDR-TB in 
Europe. A contact of some of the cases in the outbreak described in this paper became the index case of another outbreak of MDR-TB in a London hospital. ${ }^{14}$

The study hospital has 500 beds divided into eight separate buildings including two infectious disease units for male and female patients, respectively. The HIV-infected patients hospitalized in the Infectious Disease Unit are largely injecting drug users from the urban residential areas of Lisbon City within a 10-mile radius of the hospital. The hospital cares for $30 \%$ of the Lisbon HIV-infected population with 300 AIDS patients and approximately $2000 \mathrm{HIV}$-infected patients registered from 1990 to 1997.

In 1995, Infectious Disease physicians were concerned at the growing number of TB cases among HIV-infected patients attending the infectious disease unit. Of further concern was the poor clinical response observed to standard triple-drug therapy; rifampicin, isoniazid and pyrazinamide, as evidenced by a rapid progression to death, despite a moderately high CD4 count and the absence of other AIDS defining illnesses. With a view to understanding the high incidence of $\mathrm{TB}$ and poor outcome of patients with TB in this population, an epidemiological investigation and molecular study was conducted.

\section{Materials and methods}

\section{Bacteriology methods}

A case of TB was defined as a patient attending the hospital for inpatient or outpatient care from January 1993 to December 1996 with newly diagnosed positive culture TB. HIV infections were documented by demonstration of HIV antibody by enzyme-linked immunosorbent assay and confirmed by western blot analysis.

Laboratory records from January 1993 to December 1996 were reviewed and all cases of MDR-TB among HIV-infected patients recorded. Only isolates from 1995 and 1996 were available for restriction fragment length polymorphism (RFLP) DNA fingerprinting and had full susceptibilities recorded..$^{15}$

Susceptibility testing was performed using the BACTEC radiometric method for rifampicin, isoniazid, pyrazinamide, ethambutol and streptomycin. ${ }^{15}$ Breakpoints for resistance were defined as follows: isoniazid $0.1 \mu \mathrm{g} / \mathrm{mL}$; rifampicin $2.0 \mu \mathrm{g} / \mathrm{mL}$; ethambutol $7.5 \mu \mathrm{g} / \mathrm{mL}$; pyrazinamide $100 \mu \mathrm{g} / \mathrm{mL}$; and streptomycin $4.0 \mu \mathrm{g} / \mathrm{mL}$. Multidrug-resistance was defined as resistance to at least isoniazid and rifampicin.

\section{DNA fingerprinting}

\section{Primary typing}

All available isolates were subjected to IS6110 RFLP DNA fingerprinting according to standard methodology. ${ }^{16}$ Results were scanned and analysed on Gelcompar windows Version 4.0 (Applied Maths, Kortrijk, Belgium). Restriction fragment length polymorphism DNA fingerprint clustering was defined as a group of strains that differed by the presence or absence of one IS6110 band.

\section{Secondary typing}

All isolates with matching DNA fingerprints by IS6110 RFLP and those with fingerprints that differed by the presence or absence of one or two IS6110 bands were analysed using another RFLP method, polymorphic GC-rich repetitive sequences (PGRS) typing according to standard methodology. ${ }^{17}$ Results were visualized manually against a light source.

\section{Epidemiology methods}

An epidemiological investigation of a convenience sample of cases with MDR-TB was carried out by medical record review. The study group comprised those cases whose laboratory, clinical and outpatient records were available and complete from 1995 through 1996.

\section{Environmental assessment}

A general environmental assessment of the infectious diseases unit was performed. No formal smoke testing experiments were performed.

\section{Intervention}

A number of infection control measures were introduced in October 1996. All patients suspected of having infectious TB were isolated in single rooms without mechanical ventilation, starting in November 1996, then later isolated in negative pressure isolation rooms when they became available in November 1997. From November 1996, upon suspicion of TB, HIV-infected patients were 
commenced on empirical expanded drug regimens using 6-7 drugs until drug susceptibility results were available. Doctors and nurses were informed of the high risk of MDR-TB among these patients and the early and judicious use of second line drug therapy in patients not responding to the first line therapy was advised. The number of incident cases of MDR-TB among HIV-infected patients following these interventions was examined from 1997-1999.

\section{Data analysis}

Data analysis was performed using EpiInfo 6.04.18 Survival analysis was performed using statistical software for social sciences (SPSS 7.5 for windows).

\section{Results}

\section{Patient characteristics}

Thirty-five (95\%) of the 37 cases selected for epidemiological study were Portuguese; one from Africa and one from the Cape Verde Islands (Table I). All cases were male and HIV-infected (Table I). Injecting drug use was the main risk factor for HIV infection in all cases. TB was the first AIDSdefining illness in $27(73 \%)$ cases. The mean time from HIV infection to diagnosis of TB was 3.6 years. Fourteen cases (38\%) reported a history of previous treatment for TB. Information on previous treatment regimens and adherence was not available.

Table I Patient characteristics

\begin{tabular}{lll}
\hline Variable & Category & Cases $(\mathrm{N}=37)$ \\
\hline Age (years) & $20-29$ years & $15(41 \%)$ \\
& $30-39$ years & $17(46 \%)$ \\
Ethnic Group & $40+$ years & $5(14 \%)$ \\
& Portuguese & $35(95 \%)$ \\
HIV & Black African & $2(5 \%)$ \\
IVDU & Sero-positive & $37(100 \%)$ \\
& Yes & $37(100 \%)$ \\
Previous TB & No & 0 \\
& Yes & $14(38 \%)$ \\
Clustered & No & $23(62 \%)$ \\
& Yes & $31(84 \%)$ \\
Admission to IDU & No & $6(16 \%)$ \\
in previous 6 months & Yes & $36(97 \%)$ \\
\hline
\end{tabular}

IVDU, injecting drug user; IDU, Infectious Diseases Unit of Hospital (HIV unit).
All but one case (97\%) had been admitted to the male infectious disease unit in the 6 months before diagnosis of MDR-TB. The majority (80\%) of cases were exposed to multiple smear positive cases with MDR-TB during admission. Duration of exposure ranged from one day to several months. The one case not admitted overnight to the HIV unit had been on the ward for several hours awaiting medical examination, following which he was discharged home. It is very likely that he had direct contact on the open ward with two to five smearpositive patients with MDR-TB that day.

All the patients with MDR-TB died, the mean survival time after diagnosis was 83 days (range, 1-390 days, SD 89). Susceptibility results of $M$. tuberculosis isolates were not available to physicians before the death of $60 \%$ of patients.

\section{Laboratory susceptibility results}

Multidrug-resistant M. tuberculosis isolates had five main patterns of resistance listed in Table II and some varied independently of the DNA fingerprint pattern. The number of new MDR-TB cases (11 in 1993 vs. 54 in 1996) increased each year of the study period until 1997 (Table III). There was a significant decrease in the number of new cases in 1997 (54 cases in 1996 vs. 23 cases in 1997, $P<0.05)$ which continued to decrease over the next two years to the most recent figure of 16 cases in 1999 (Fig. 1).

Table II Pattern of drug susceptibility in multidrug-resistant tuberculosis isolates among HIV infected patients attending the infectious diseases unit January 1995 to December 1996

\begin{tabular}{l} 
Susceptibility patterns \\
\hline Isoniazid, rifampin and ethambutol \\
Isoniazid, rifampin, ethambutol and pyrazinamide \\
Isoniazid, rfiampin and streptomycin \\
Isoniazid, rifampin, streptomycin, ethambutol and pyrazinamide \\
Isoniazid, rifampin streptomycin, and pyrazinamide
\end{tabular}

Table III Drug susceptibility testing result on Mycobacterium tuberculosis isolates among HIV infected patients January 1993 to December 1996

\begin{tabular}{lcc}
\hline & $\begin{array}{l}\text { Fully susceptible } \\
\text { M. tuberculosis }\end{array}$ & MDR-TB \\
\hline 1993 & 20 & 11 \\
1994 & 24 & 21 \\
1995 & 34 & 40 \\
1996 & 30 & 54 \\
\hline
\end{tabular}




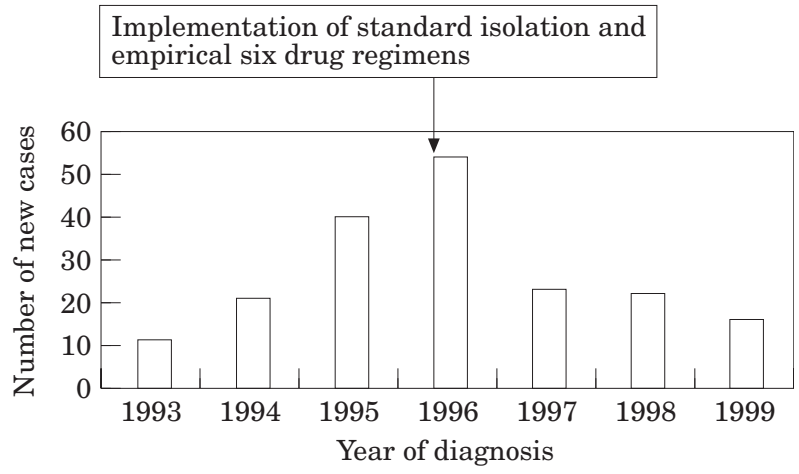

Figure I Cases of Multidrug-resistant TB among HIV-infected patients treated at the study hospital Lisbon 1993-1999. Initiation of standard isolation and 6 drug therapy demonstrated by arrow.

\section{Strain typing}

IS6110 RFLP typing was performed on 134 M. tuberculosis isolates obtained from the hospital laboratory during the four years of the study, 95 of these were MDR and the remaining 39 were fully drug-susceptible. All 39 drug-susceptible isolates had a unique pattern on DNA fingerprint, whereas 72 of the $95(76 \%)$ MDR-TB isolates were clustered into two main clusters, A and B. Thirty-five $(36 \%)$ of the MDR-TB isolates were in cluster A, and $37(39 \%)$ were in cluster B. The thirty-five isolates in cluster $\mathrm{A}$ had the exact same nine band pattern (Fig. 1). Isolates in cluster B pattern demonstrated two sub-clusters, 22 of cluster B isolates had 11 bands and 15 had an additional band. Overall there was considerable similarity in RFLP DNA fingerprint patterns between the two main clusters, cluster $\mathrm{A}$ and cluster $\mathrm{B}$ both sharing nine common bands (Fig. 2). Twenty-three remaining MDR isolates fell into three smaller clusters, C, D and $\mathrm{E}$.

Over the four-year period that isolates were collected, the predominant fingerprint pattern changed. Cluster pattern A accounted for $70 \%$ of MDR-TB isolates in 1993; this decreased to $15 \%$ in 1996. Cluster pattern B appeared in 1995 and became the dominant pattern representing 63\% of MDR-TB isolates in 1996 (Fig. 3). Cluster A and cluster B were indistinguishable by secondary typing using PGRS, ${ }^{17}$ demonstrating that these isolates were highly genotypically related.

\section{Environmental assessment prior to interventions}

The male infectious diseases unit had four main open bay wards with six beds in each ward and two

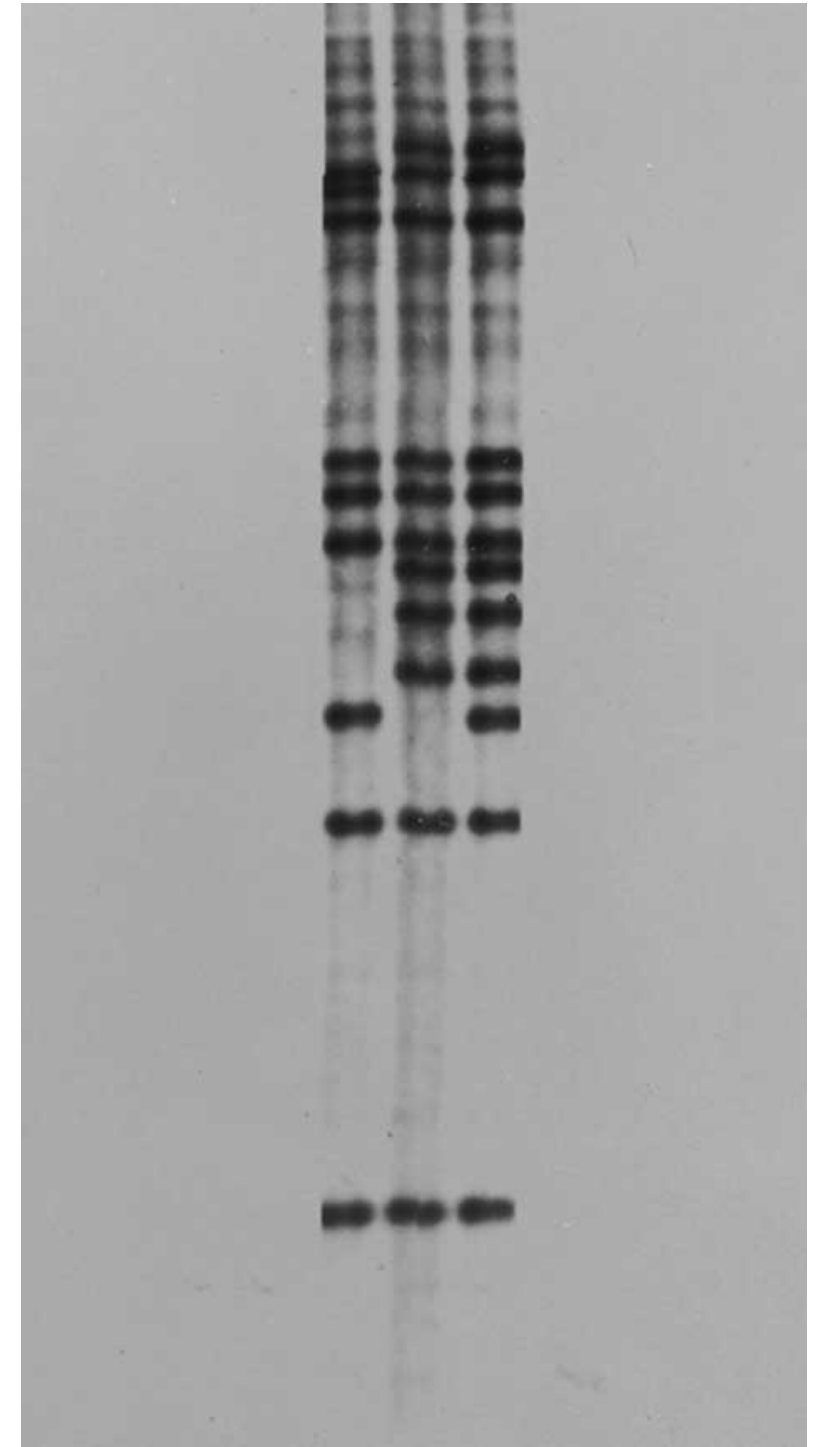

Figure 2 DNA Fingerprints patterns of Mycobacterium tuberculosis isolates belonging to the following sub-clusters, $\mathrm{A}, \mathrm{BI}$, and $\mathrm{B} 2$.

side rooms, each containing one bed. The open bay wards and side rooms were not mechanically ventilated, and during the summer, air was circulated between the corridors and the wards by small ceiling fans and open windows.

\section{Infection control interventions}

In November 1996 all HIV patients admitted to the HIV unit suspected with TB were isolated in side rooms. Patients were instructed not to leave their rooms and staff entering and leaving the rooms observed standard isolation procedures, including the wearing of standard surgical masks. One year 


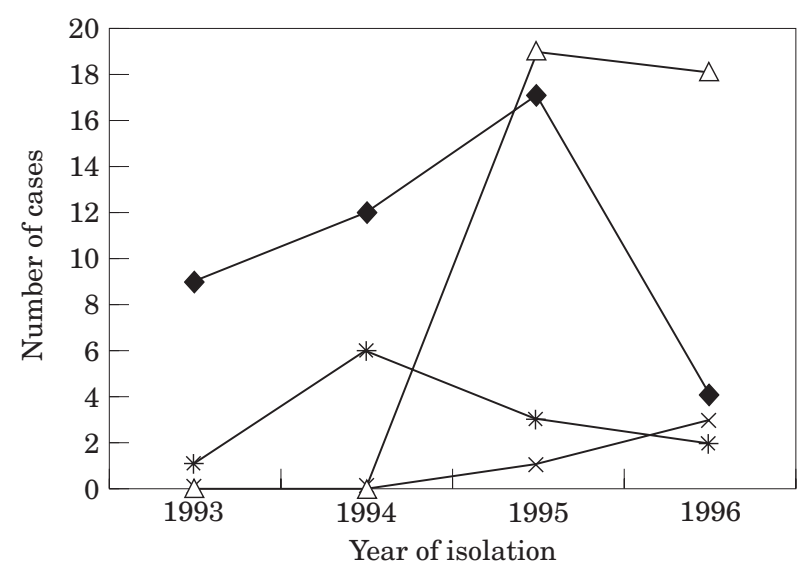

Figure 3 Year of isolation of MDR M. tuberculosis strains with genotypically related DNA fingerprint pattern $A$ and pattern $B$, subclones $\mathrm{BI}$ and $\mathrm{B} 2$ during the course of the outbreak. $\longrightarrow \mathrm{A}, 9$ bands; $-\mathrm{B}, \mathrm{II}$ bands; $\rightarrow \mathrm{BI}, 12$ bands; $\rightarrow$, unique.

later, four negative pressure rooms had been constructed and were used in addition to side rooms to house infected TB patients. Air was ventilated to the outside in each case and rooms were maintained under negative pressure at all times.

\section{Outcome of intervention}

The outcome of improved infection control practices and empirical use of second line drug therapy was that the number of new cases of MDR-TB among the HIV-infected patients sharply declined from 54 cases in 1996 to 23 in 1997. There was no evidence, however, that the introduction of negative pressure ventilation had any further impact on reducing transmission when introduced one year later (Fig. 1).

\section{Discussion}

Over three decades in Portugal, the incidence of TB steadily declined. Since 1991, however there has been a reversal of this downward trend and a slight increase in the reported incidence of TB, particularly in the coastal region (Lisbon and Porto) of Portugal. ${ }^{19}$ In 1995, the Portuguese Health Authorities reported a TB incidence of 49.8/100000 population nationwide and $61.7 / 100000$ in the city of Lisbon. ${ }^{20}$ Lisbon City is most affected by the dual pandemic of HIV and TB and in 1995, an estimated $15 \%$ of $\mathrm{HIV}$-infected patients developed TB. The increase was particularly apparent in the 15-34 year age group and associated with male gender, HIV infection, injecting drug use and a high mortality. In spite of the high incidence of $\mathrm{TB}$, the national anti-TB control program was discontinued and as a consequence, countrywide data on drugsusceptibility is not available.

The reported rate of $\mathrm{MDR}$ among $\mathrm{TB}$ cases in Portugal was $3.5 \%$ in 1994. Preliminary results of a prospective study in 1997 have identified an increase to $4.3 \%$ (pers. comm., Dr A. F. Antunes). These national figures are significantly lower than those found in this study where $41 \%$ of $\mathrm{TB}$ isolates at a Lisbon city hospital in 1996 were found to be MDR. More recently other hospitals in Lisbon have seen an increase in the number of cases of MDR-TB among HIV-infected patients. ${ }^{21}$

In this study, we conducted an outbreak investigation of MDR-TB among male HIV-infected patients attending a hospital in Lisbon. We carried out a retrospective molecular epidemiological study on a proportion of these cases and re-evaluated the situation in the ensuing years to determine whether control measures had reduced nosocomial transmission of infection. The epidemiological and molecular investigation of this outbreak provides evidence that there was nosocomial transmission of infection with MDR-TB among these patients for the following reasons. Firstly, $80 \%$ of case patients were documented to have had contact with infectious patients in hospital; secondly, incubation periods from time of exposure to development of active disease were consistent with those observed in previous nosocomial outbreaks among AIDS patients, ${ }^{2-24}$ thirdly, isolates had matching RFLP DNA fingerprints patterns, and finally, examination of ward facilities at the time of suspected transmission identified the absence of any isolation rooms or air handling systems which might have protected these immunocompromised patients from infectious aerosols.

One patient with a clustered isolate had no documented admission to the infectious diseases ward prior to his MDR-TB diagnosis, but had attended the HIV outpatient clinic on several occasions. On one of the occasions the patient had spent several hours on the HIV ward awaiting medical examination. At this time there were five other patients with smear positive-pulmonary MDR-TB on the open ward who the patient had contact with. Epidemiological investigation of this outbreak was limited because patients had died before the study was begun. Given, however, that these cases were all between 20 and 39 years, injecting drug users and HIV-infected, it is possible they had some contact outside the hospital and that some transmission may have occurred in the community. 
In Lisbon, patients with $\mathrm{TB}$ do not receive supervised treatment, i.e., Directly Observed Therapy Short course (DOTS). ${ }^{25}$ The absence of supervised therapy in this population may result in poor treatment compliance and the development of drug resistance. The absence of a ventilation system that re-circulates or removes air and/or isolation rooms on the HIV wards, meant that infectious cases shared open wards with other highly immunocompromised patients and this is likely to have contributed to transmission of infection. Other factors such as delays in recognizing drug resistance, and initiating appropriate treatment meant that patients with MDR-TB were treated with inadequate regimens and probably remained highly infectious during admission or until death.

The high degree of similarity between IS6110 RFLP DNA fingerprint patterns in the A and $\mathrm{B}$ clusters and the fact that they are indistinguishable using PGRS typing suggests that they were closely related genetically. The results of this study suggest the spread of a closely related family of MDR-TB strains that have shared common ancestry and have undergone clonal expansion. This is similar to the situation in New York where strain $\mathrm{W}$ was found to account for a high proportion of cases of MDR-TB. ${ }^{5,26,27}$

The limitations of this study are that direct contact to an infectious case could not be linked to any one patient as there were usually several infectious cases present on the ward at any one time and also that not all cases were available for epidemiological investigation. Molecular analysis using PGRS confirmed the genotypic relatedness of these strains and further work found one common rpo B mutation among the majority of these strains, again suggesting they are genotypically highly related. The chronology of TB demonstrated in the patients under study suggests that pattern A was the original clone seen in 1993 and that pattern B appeared later as a result of genetic shift creating two genetically related clusters. Relating this to the clinical setting, from what we know of the conserved nature of the TB genome; it is likely that what we are seeing here is not a single outbreak with a rapidly evolving fingerprint, but several smaller overlapping outbreaks at the same time and place with strains that were once directly related. ${ }^{28,29}$

There are several similarities between the TB situation in Lisbon and that seen in New York in the late 1980s and early 1990s. Delayed recognition of MDR-TB means that patients are inadequately treated and remain highly infectious for prolonged periods. Inadequate isolation facilities in hospitals treating immunocompromised HIV-infected patients allows the potential for rapid spread of M. tuberculosis.

The most important interventions enabling the epidemic to be controlled in New York were widespread introduction of DOTS, improved hospital infection control, including extensive use of negative pressure isolation facilities, increased index of suspicion of $\mathrm{TB}$, early diagnosis of $\mathrm{TB}$, and more timely culture and susceptibility results. ${ }^{30}$ This study shows that the level of MDR-TB decreased following introduction of standard isolation side rooms without negative pressure ventilation, along with the initiation of a 6 or 7 drug combination on all patients, whilst awaiting susceptibility results. The later introduction of negative pressure did not result in further reduction of MDR-TB. Our data therefore, suggest that priority should be given to standard isolation rooms and the provision of second-line anti-TB drugs above the provision of negative pressure isolation rooms, should budget constraints make such a choice necessary.

\section{Acknowledgements}

We are indebted to the staff of the Curry Cabral Hospital who made this study possible; to Ann Dickens, Department of Medical Microbiology, Royal Free Hospital, London, for help with DNA fingerprinting; to Richard Arnold, Caroline Baker and Ann Grossinho, Department of Epidemiology and Public Health, Imperial college School of Medicine for statistical analysis; to $\mathrm{Dr}$ David Hawkins for his support. The authors are grateful to Dr Renee Ridzon, Division of TB Elimination, Centers for Disease Control, Atlanta, Georgia, US and Dr Antonio Fonseca Antunes, General Director of health, Portugal for their valuable contribution to the manuscript.

\section{References}

1. Dye C, Scheele S, Dolin P, Pathania V, Raviglione MC. Consensus statement. Global burden of tuberculosis estimated incidence, prevalence, and mortality by country. WHO Global Surveillance and Monitoring Project. $\mathcal{F} A M A$ 1999; 282: 677-686.

2. Raviglione M, Snider D and Kochi A. Global epidemiology of tuberculosis. Morbidity and mortality of a worldwide epidemic. FAMA 1995; 273: 220-226. 
3. Beck-Sague CM, Dooley SW, Hutton MD et al. Hospital outbreak of multidrug-resistant Mycobacterium tuberculosis infection. FAMA 1992; 268: 1280-1286.

4. Pearson ML, Jereb JA, Frieden TR et al. Nosocomial transmission of multidrug-resistant mycobacterium tuberculosis: a risk to patients and health care workers. Ann Intern Med 1992; 117: 191-196.

5. Coronado VG, Beck-Sague CM, Hutton MD et al. Transmission of multidrug-resistant Mycobacterium tuberculosis among persons with human immunodeficiency virus infection in an urban hospital: epidemiological and restriction length polymorphism analysis. F Infect Dis 1993; 168: 1052-1055.

6. Valway S, Griefinger R, Papania M et al. Multidrugresistant tuberculosis in a New York State Prison System, 1990-1991. F Infect Dis 1994; 170: 151-156.

7. Valway SE, Richards S, Kovacovich J, Griefinger R, Crawford J, Dooley SW. Outbreaks of multidrugresistant tuberculosis in a New York State Prison, 1991. Am ₹ Epidemiol 1991; 140: 113-122.

8. Jereb JA, Klevens M, Privett T et al. Tuberculosis in health care workers at a hospital with an outbreak of multidrug-resistant Mycobacterium tuberculosis. Arch Intern Med 1995; 155: 854-859.

9. Agerton T, Valway SE, Gore B, Pozsik C, Bond W, Onorato I. Transmission of a highly drug-resistant strain (strain W) of Mycobacterium tuberculosis; community outbreak and nosocomial transmission via a contaminated bronchoscope. $\mathcal{F} A M A$ 1997; 278: 1072-1077.

10. Guerrero A, Cobo J, Fortun J et al. Nosocominal transmission of Mycobacterium bovis resistant to 11 drugs in people with advanced HIV-infection. Lancet 1997; 350: 1738-1742.

11. Rullan JV, Herrera D, Cano R et al. Nosocomial transmission of multi drug-resistant Mycobacterium tuberculosis in Spain. Emerg Infect Dis 1996; 2: 125-129.

12. Bouvet E, Casalino E, Mendoza-Sassi G et al. A nosocomial outbreak of multidrug-resistant Mycobacterium bovis among HIV-infected patients. A case-control study. AIDS 1993: 7: 1453-1460.

13. Moro LM, Gori A, Errante I et al. and the Italian Multidrug-resistant tuberculosis Outbreak Study Group. An outbreak of multidrug-resistant tuberculosis involving HIV-infected patients of two hospitals in Milan, Italy. AIDS 1998; 12: 1095-1102.

14. PHLS/CDSC: Outbreak of hospital-acquired multidrug-resistant tuberculosis. Commun Dis Rep Wkly 1995; 5: 161.

15. Roberts GD, Goodman NL, Heifets $\mathrm{L}$ et al. Evaluation of the BACTEC radiometric method for recovery of mycobacteria and drug susceptibility testing of Mycobacterium tuberculosis from acid-fast smear-positive specimens. F Clin Microbiol 1983; 18: 689-696.

16. van Sollingen D, de Haas PEW, Hermans PWM, van Emden JDA. Protocol RFLP analysis of
Mycobacteria. Protocol MMB, National Institute Public Health and Environmental Protection. Bilthowen, Netherlands, 1993.

17. Ross BC, Raios K, Jackson K, Dwyer B. Molecular cloning of a highly repeated element from Mycobacterium tuberculosis and its use as an epidemiological tool. F Clin Microbiol 1992; 30: 942-946.

18. Dean AG, Dean JA, Coulombier D et al. Epi Info, Version 6: a word processing, database, and statistical program for public health on IBM-compatible microcomputers. Centers for Disease Control and Prevention. Atlanta, Georgia, USA, 1995.

19. Antunes ML, Fonseca-Antunes A. The tuberculosis situation in Portugal: a historic perspective to 1994. EuroSurveillance. 1996; 1; 19-21.

20. Tuberculose em Portugal. Statistics and Epidemiology. Director of Services of Public Health. Lisbon, Portugal, 1996.

21. Portugal I, Covas MJ, Brum L et al. Outbreak of multiple drug-resistant tuberculosis in Lisbon: detection by restriction fragment length polymorphism analysis. Int $\mathcal{f}$ Tuberc Lung Dis 1999; 3: 207-213.

22. Fischl MA, Uttamchandani RB, Daikos GL et al. An Outbreak of tuberculosis caused by multidrug-resistant bacilli among patients with HIV Infection. Ann Intern Med 1992; 117: 177-183.

23. Dooley SW, Villarino ME, Lawrence M et al. Nosocomial transmission of tuberculosis in a hospital unit for HIV-infected patients. $\mathcal{F A M A} 1992 ; 267$ : 2632-2634.

24. Edlin BR, Tokars JI, Grieco MH et al. An outbreak of multidrug-resistant tuberculosis among hospitalised patients with the acquired immunodeficiency syndrome. N Engl F Med 1992; 326: 1514-1521.

25. Sumartojo E. When tuberculosis treatment fails. A social behavioral account of patient adherence. Am Rev Respir Dis 1993; 147: 1311-1320.

26. Bifani P, Plikaytis B, Kapur V et al. Origin and interstate spread of a New York City multidrug-resistant Mycobacterium tuberculosis clone family. FAMA 1996; 275: 452-457.

27. Friedman CR, Stoeckle MY, Kriswirth BN et al. Transmission of multidrug-resistant tuberculosis in a large urban setting. Am 7 Respir Crit Care Med 1995; 152: 355-359.

28. Kubin M, Havelkova M, Hyncicova I et al. A multidrug-resistant tuberculosis microepidemic caused by genetically closely related Mycobacterium tuberculosis strains. F Clin Microbiol 1999; 37: 2715-2716.

29. Portugal I, Maia S, Moniz-Pereira J. Discrimination of multidrug-resistant Mycobacterium tuberculosis IS6110 fingerprinting subclusters by rpoB gene mutation analysis. F Clin Microbiol 1999; 37: 3022-3024.

30. Frieden TR, Fujiwara PI, Washko RM, Hamburg MA. Tuberculosis in New York City - turning the tide. N Engl F Med 1995; 333: 229-333. 